\title{
Zur brasilianischen Textart "Memorial Acadêmico" - wie man sich in Brasilien auf eine akademische Stelle bewirbt
}

\author{
Kathrin Schweiger
}

\begin{abstract}
This paper analyses the Brazilian genre "Memorial acadêmico" and its function, outlining similarities and differences in comparison with the German Genre "Akademischer Lebenslauf. Based on a corpus of seven "Memoriais", differences in terms of proposition and illocution between these two genres were identified.
\end{abstract}

Key-words: Genre in Academic Setting, Autobiography, Contrastive Analysis of Genres.

Resumo: O presente trabalho tem como objetivo analisar o gênero "Memorial Acadêmico" e as suas funções, propõe também uma discussão entre as semelhanças e diferenças entre esse gênero e o gênero "Akademischer Lebenslauf", usual na Alemanha. O estudo baseou-se em seis Memoriais nos quais podem-se constatar as diferenças do gênero tanto na proposição quanto na ilocução, assim como o seu uso nos respectivos países.

Palavras-chaves: Gênero acadêmico, Autobiografia, Análise Contrastiva de Gêneros Acadêmicos.

Zusammenfassung: Um sich in Brasilien auf eine wissenschaftliche Stelle zu bewerben, muss oftmals ein "Memorial Acadêmico" eingereicht werden. Eine Textart, die es so im deutschsprachigen Raum nicht gibt. Eine in funktionaler Hinsicht ähnliche Textart liegt im "Akademischen Lebenslauf" vor. In diesem Artikel sollen anhand eines Korpus von sechs "Memoriais" Unterschiede und Gemeinsamkeiten in Illokution und Proposition dieser beiden Textarten aufgezeigt werden.

Stichwörter: Akademischer Text; Autobiografie; Kontrastive Analyse akademischer Textarten.

\footnotetext{
* Doutoranda do Programa de Pós-graduação em Língua e Literatura Alemã da Universidade de São Paulo. Email: schweiger@usp.br
} 
Schweiger, K. - Zur brasilianischen Textart "Memorial Acadêmico"

\section{O. Einleituns}

Wenn man sich in Brasilien auf eine wissenschaftliche Stelle bzw. auf eine Dozentenstelle an der Universität bewirbt, muss man ein so genanntes Memorial Acadêmico einreichen. Zunächst einmal findet sich in Deutschland bzw. im deutschsprachigen Raum keine dem brasilianischen Memorial eins zu eins entsprechende Textart ${ }^{1}$. Grundsätzlich entspricht diese Textart aber hinsichtlich ihrer Funktion dem in Deutschland geläufigen Akademischen Lebenslauf. Bevor der Wissenschaftler eine neue Stelle an einer Universität antritt, muss er sich normalerweise einem Auswahlverfahren unterziehen; dieses beginnt schon mit dem Abfassen und Einreichen des Lebenslaufes. Zwischen der brasilianischen Version und der deutschen bestehen jedoch große Unterschiede bezüglich Proposition (Inhalt), Illokution (Sprechhandlungen) und Stil. Diese Unterschiede sollen in der vorliegenden Arbeit dargestellt werden. Es zeigt sich, dass es sich beim Memorial Acadêmico nicht um eine verschiedensprachige Version eines akademischen Lebenslaufes handelt, sondern um eine in jeder Beziehung andersartige Textart. Als Korpus dienen sechs Exemplare der Textart Memorial, die bei Bewerbungen um Dozentenstellen der Literatur-, Sprach- und Geschichtswissenschaft vorgelegt wurden ${ }^{2}$.

\section{Zur kulturellen Bedingtheit von Texten}

Es wird davon ausgegangen, dass Textarten kulturell geprägt sind, d.h., die Denkweisen, Vorstellungen und Verhaltensweisen einer Gesellschaft X spiegeln sich in ihnen

\footnotetext{
1 „Eine Textart ist eine zweckbezogene Tiefenstruktur, die spezifische kommunikative Aufgaben zu bearbeiten gestattet, denen repetitiv immer neue Handelnde gegenüberstehen. Textarten sind also Großmuster des sprachlichen Handelns, denen eben jener Ressourcencharakter zukommt, der für die Bearbeitung gesellschaftlicher Zwecke unumgänglich ist (EHLICH 2000:11)“. Zentral für Ehlichs Textdefinition ist der gesellschaftliche Zweck, das heißt, dass der spezifische Zweck einer Textart $\mathrm{X}$ in einen größeren gesellschaftlichen Zusammenhang eingebunden ist - hier in die Institution der Wissenschaft; der Universität und ihren zugehörigen Einrichtungen.

2 Die Memoriais sind grundsätzlich der Öffentlichkeit zugänglich, dies entspricht dem transparenten Bewerbungsprozess in Brasilien. Dennoch handelt es sich m. E. um eine sehr persönliche Textart, sodass die Namen der Verfasser nicht direkt genannt werden. Die Memorias stammen aus den Universitäten UNESP Assis (zwei Memoriais eingereicht bei der Französischfakultät 1989, Theoretische Literatur 1987), UNESP Arararaquara (Deutschfakultät 2002, Italienischfakultät 2002), UNESP São José de Rio Preto (Französischfakultät 1993), USP São Paulo (Fakultät für Geschichte, 1987).
} 


\section{Schweiger, K. - Zur brasilianischen Textart "Memorial Acadêmico"}

wider (REHBEIN 1985: 30). Dabei ist Kultur das Resultat einer historisch gewachsenen Entwicklung, bei der Sprache eine mediale Funktion zukommt. Zudem manifestieren sich in ihr die kulturellen Eigenheiten einer Gemeinschaft, d.h., die sprachlichen Ausdrucksformen werden durch Kultur geprägt (a.a.O.). ${ }^{3}$

Textarten, denen der gleiche Zweck zugrunde liegt, die aber aus unterschiedlichen Kulturkreisen kommen, können daher Unterschiede in ihren Textmustern aufweisen. Diese Unterschiede können maßgeblich dazu beitragen, dass die Texte nicht als Textart als solche begriffen werden. Nach ESSER (1997) sind diese Unterschiede sowohl bei der sprachlichen Darstellung als auch bei der strukturellen Aufmachung der Texte auszumachen. Dabei weist sie ausdrücklich darauf hin, dass man bei einem Vergleich der Texte es nicht bei einer kontrastiv sprachlichen Untersuchung belassen darf. Vielmehr müssen die Texte in einen „kulturellen Bezugsrahmen“ (ESSER 1997:35) gestellt werden. Demnach bestimmen „(...) außertextuelle ${ }^{4}$, kulturbezogene Faktoren das Textmuster einer Textsorte, also ihre inhaltliche, strukturelle, sprachliche und funktionale Gestaltung (...)“(ebd.).

Ich gehe davon aus, dass ein Memorial acadêmico und ein akademischer Lebenslauf die gleiche Funktion aufweisen: sich bewerben um eine Stelle. Bei beiden geht es darum, seine Lebensstationen in einer für den potenziellen „Arbeitgeber“ relevanten Darstellung offen zu legen. Allerdings zeigt sich schon in der Bezeichnung ,relevant“ die kulturelle Dimension beim Verfassen dieser Textart. Im Folgenden möchte ich anhand von Auszügen aus den mir vorliegenden „Memoriais“ funktional-pragmatische sowie kulturelle Eigenschaften dieser Textart aufzeigen. Zunächst soll aber unter Punkt zwei noch eine etymologisch-definitorische Skizzierung der Begriffe Biografie, Autobiografie und Memoiren vorgenommen werden.

\footnotetext{
${ }^{3}$ Nach KAISER (2002) stellt sich dabei aber zu Recht die Frage, inwieweit sprachliche Ausdrucksformen ihrerseits wiederum die kulturelle Dimension prägen.

4 “außertextuell” meint nach EHLICH (2000) den Zweck.
} 
Schweiger, K. - Zur brasilianischen Textart "Memorial Acadêmico"

\section{Biografie - Autobiografie - Memoiren - Memorial Acadêmico}

Die Begriffe Memorial/Memoiren gehen auf das lateinische Wort memoria ${ }^{5}$ (Erinnerung, Gedenken) zurück. Wenn wir im Deutschen von Memoiren sprechen, werden diese dabei oftmals mit der Textart Autobiografie verwechselt. Eine Autobiografie ist eine Biografie (von griech. bios - Leben; grafein - schreiben), die von einer Person selbst (auto) geschrieben wird (MISCH 1989), während die Biografie das Leben einer Person beschreibt, ohne dass es eine Übereinstimmung zwischen Autor und Protagonisten der Handlung gibt. LEHMANN (1988: 36) definiert die Autobiografie aus pragmatischer Perspektive als eine „(...) Textart, durch die ihr Autor in der Vergangenheit erfahrene innere und äußere Erlebnisse sowie selbst vollzogene Handlungen in einer das Ganze zusammenfassenden Schreibsituation sprachlich in narrativer Form so artikuliert, daß er sich handelnd in ein bestimmtes Verhältnis zur Umwelt setzt“. BRUSS (1989: 273) spricht von „Regeln für den autobiografischen Akt“. Der Autor verpflichtet sich zur Wahrheit seiner Geschichte, während der Leser sowohl die ihm präsentierten Ereignisketten als auch die Identität zwischen Autor, Erzähler und Protagonisten als Faktum anerkennt. Wie schon erwähnt, wird die Textart der Memoiren häufig unter die oben genannte Definition von Misch subsumiert; literaturwissenschaftlich betrachtet unterscheiden sich aber die Memoiren und die Autobiografie in einem Punkt. Während die Autobiografie die Entwicklung einer individuellen Persönlichkeit zu rekonstruieren versucht, das heißt, den Menschen von Anfang an bis hin zu seiner Sozialisierung unter Augenschein nimmt, befassen sich Memoiren ${ }^{6}$ mit dem Werdegang einer Person im Rahmen ihres gesellschaftlichen Umfelds. Damit ist gemeint, dass die soziale Funktion der Person im Mittelpunkt steht wie z.B. beruflicher Werdegang, politische Karriere, das Schaffen als Künstler etc. Des Weiteren unterscheiden sich die beiden Textarten hinsichtlich des Zeitrahmens. Memoiren beschreiben oftmals nur einen Lebensabschnitt,

\footnotetext{
${ }^{5}$ PRADO (2006) listet für den Begriff memória im Portugiesischen 11 Bedeutungsmöglichkeiten auf:

1. faculdade de reter as idéias, impressões e conhecimentos adquiridos anteriormente 2. lembrança, reminiscência, recordação 3. celebridade, fama, nome 4. monumento comemorativo 5. relação, relato, narração 6. memento 7.vestígio, sinal 8. nota diplomática 9. dissertação acerca de assuntos diversos 11. dispositivo do computador 12. poder criador, imaginação, talento.

${ }^{6}$ Im Portugiesischen findet man die Pluralform memórias als Begriffsbezeichnung neben memorial. Mir scheint, dass memórias der generelle Begriff für Memoiren sind und memorial acadêmico die spezifische Bezeichnung für die Textart im Sinne eines akademischen Lebenslaufs ist.
} 


\section{Schweiger, K. - Zur brasilianischen Textart "Memorial Acadêmico"}

während die Autobiografie das gesamte Leben schildert. Auch in Brasilien unterscheiden sich memorial und autobiografia in diesem Punkt (RAMOS/GoNCALVES 1996:6).

(...) [o autor] faz um relato de sua própria vida, procurando apresentar acontecimentos a que confere o status de mais importantes, ou interessantes, no âmbito de sua existência. Possivelmente, ele só levantará o véu apenas da parte que, de si próprio, pretende que se saiba e que se venha a ser lembrada. ${ }^{7}$

Anders als bei der Biografie gibt es keine "Verpflichtung" das ganze Leben zu schildern (PRADO 2006).

Die diffuse Verwendung der Begriffe Autobiografie/Memoiren kann auch zum einen daran liegen, dass der Begriff Memoiren lange Zeit für die Textart Autobiografie verwendet wurde, während der Ausdruck Autobiografie nach MISCH (1989) erst im 19. Jahrhundert im deutschsprachigen Raum Einzug fand und den Memoiren-Begriff ablöste. „Dieser Ausdruck hat einen tieferen Sinn insofern, als er, aus dem Wort 'Erinnerung' gebildet, auf die psychologische Quelle der Geschichte - und nach der klassisch-griechischen Anschauung auch der Poesie - hinweist.“ (Misch 1989: 39ff). STEPHAN (2004) weist den Memoiren eine Zwitterstellung zwischen Faktenbericht und literarischem Kunstwerk zu. In ähnlicher Weise äußert sich WAIZBORT (1998:1), indem er schreibt :

O memorial converte o candidato em um narrador de extração distinta da que lhe é usual. Se a escrita e algumas formas narrativas lhe são familiares, em uma vida que consiste também em escrever textos, o texto do memorial impõe suas exigências peculiares e, para muitos dos seus autores mais recentes, é oportunidade para dar curso a pretensões narrativas que, no dia-a-dia da produção científica, ficam recalcadas.

Wo ist nun die brasilianische Textart Memorial zu verorten? Meines Erachtens handelt es sich hierbei um eine Mischung aus Memoiren und ausführlichem Lebenslauf. Das Memorial acadêmico beschreibt das „Werden zum Akademiker“8, während es sich beim deutschen

\footnotetext{
${ }^{7}$ Der Autor verfasst einen Bericht über sein Leben, bei dem er nur die wichtigsten und interessantesten "Ereignisse" seines Lebens heraussucht. Er hebt den Schleier nur soweit wie er meint, dass diese Begebenheiten gedenkwürdig sind.

${ }^{8}$ WAIZBORT (ebd.) verweist in diesem Zusammenhang auf den im 18. Jahrhundert entstandenen Bildungsroman.
} 
Schweiger, K. - Zur brasilianischen Textart "Memorial Acadêmico"

akademischen Lebenslauf um eine bloße Aufreihung der Fakten handelt und der reflektorische Prozess außer Acht gelassen wird.

\section{Makrostruktur}

Die makrostrukturelle Untersuchung umfasst die Analyse der Textteile und den formellen Aufbau der jeweiligen Textart allgemein. Zunächst einmal gilt es festzustellen, aus welchen Teilen sich ein Memorial zusammensetzt.

\subsection{Textteile}

Ein Memorial kann 30 bis 100 Seiten umfassen und beginnt mit einem Deckblatt, auf dem neben dem Namen des Bewerbers, dem Datum und der expliziten Erwähnung der Textart ${ }^{9}$ auch die namentliche Nennung der Institution sowie die ausgeschriebene Stelle aufgeführt ist. Es folgt ein Inhaltsverzeichnis, eine Art Einführung/Vorwort, ein Haupttext sowie der tabellarische Lebenslauf. Es findet sich hin und wieder auch nach dem Deckblatt ein Extrablatt mit einer Widmung oder einem Gedicht/ Sprichwort als Einstieg:

\section{Beispiel 1}

A meus pais, lutadores que me ensinaram a perseverança e a determinação; A Flavio, amigo e companheiro, que me acompanha e ampara há muito nessa jornada; A Pedro ${ }^{10}$ e Mariana, frutos de nosso amor e herdeiros de nossa disposição de luta.

Für meine Eltern, Kämpfer, die mich Durchhaltevermögen und Bestimmtheit gelehrt haben, für Flavio, Freund und Begleiter, der mich schon lange auf diesem Weg begleitet und unterstützt. Für Pedro und Mariana, Früchte unserer Liebe und Erben unserer Kampfbereitschaft.

\footnotetext{
9 „Die Bezeichnung eines Textes durch seinen Verfasser lässt darauf schließen, dass der Verfasser Textartbewusstsein besitzt und somit auch ,einen gewissen Formwillen [...] verrät“ (KAISER 2002:169).

${ }^{10}$ Namen wurden von der Verfasserin aus Datenschutzgründen geändert.
} 
Schweiger, K. - Zur brasilianischen Textart "Memorial Acadêmico"

\section{Beispiel 2}

Wer von sich selber zu erzählen beginnt, beginnt meist mit ganz anderen Leuten. Mit Menschen, die er nie getroffen hat und niemals treffen wird. Mit Menschen, die schon lange tot sind, und von denen er fast gar nichts weiß. Wer von sich selber zu erzählen beginnt, beginnt meist mit seinen Vorfahren.

(Erich KÄSTNER*1899/+1974-Als ich ein kleiner Junge war)

Oder es gibt einen Anhang, der dann ein Gedicht (z.T.über mehrere Seiten hinweg) enthält, das dem Bewerber besonders passend oder auf ihn zutreffend erscheint.

Bei den meisten Memoriais ist ein Inhaltsverzeichnis (portugiesisch sumário oder indice) vorangestellt, das folgende Themen beinhaltet:

Abb. 1

\begin{tabular}{|l|}
\hline $\begin{array}{l}\text { Persönliche Angaben (Name, Name der Eltern, Geburtsortdatum, } \\
\text { Ausweisnummer, Steuerzahlernummer, Wohn-/Arbeitsort, } \\
\text { Nationalität, Zivilstand, Wahlberechtigungsnummer, Nummer des } \\
\text { Arbeitsausweises usw.) }\end{array}$ \\
\hline schulischer Werdegang \\
\hline akademischer/beruflicher Werdegang \\
\hline $\begin{array}{l}\text { Auflistung der während der Mestrado-bzw. Doutoradozeit besuchten } \\
\text { Seminare }\end{array}$ \\
\hline Publikationsliste \\
\hline Projekte \\
\hline unterrichtete Fächer \\
\hline gehaltene Vorträge/Kongressteilnahmen \\
\hline „Zwischen den Zeilen“ \\
\hline Lebenslauf \\
\hline
\end{tabular}

Bei den persönlichen Daten ist festzustellen, dass es dabei sehr „bürokratisch zugeht““. Sämtliche Identifikationsnummern werden angegeben (Steuerzahlernummer, Wahlberechtigungsnummer etc.).

Es zeigt sich, dass es keine strikten formellen Vorgaben hinsichtlich des Aufbaus bzw. der Abfolge gibt. Allerdings lässt sich eine gewisse Zweigliedrigkeit feststellen, d.h. in einem ersten Teil findet sich der ,ausführliche Lebenslauf“ (eben die Memoiren) und in einem 
Schweiger, K. - Zur brasilianischen Textart "Memorial Acadêmico"

zweiten Teil der „tabellarische Lebenslauf“ (mit den Persönlichen Angaben vorangestellt) oder auch vice versa.

Abb. 2

\begin{tabular}{|c|c|}
\hline Beispiel 3 & Beispiel 4 \\
\hline Sumário & Indice \\
\hline Introdução & I. Explicação necessária \\
\hline 1. Começando... & II. Lembrança do mundo antigo \\
\hline 2. Partindo... & III. Á la recherche du temps perdu \\
\hline 3. Voltando às origens... & A. Professora-aluna/aluna-professora \\
\hline 2. E agora?... & B. Professora-pesquisadora \\
\hline$\cdots+$ & C. Pausa \\
\hline 9. Agora, podendo trabalhar mais com a & D. Retomando a narrativa \\
\hline docência, podendo me dedicar mais aos alunos... & E. De como não fui professora da USP \\
\hline 10. E o doutorado? & IV. Le temps retrouvé \\
\hline Curriculum vitae & A. A docência \\
\hline 1. Dados Pessoais & B. A pesquisa \\
\hline 2. Formação & C. A prestação de serviços à communidade \\
\hline \multicolumn{2}{|l|}{ 2.1. Formação Básica } \\
\hline 2.2. Formação Universitária & V. Considerações finais \\
\hline 3. Atividades de Formação & VI. Curriculum vitae \\
\hline \multicolumn{2}{|l|}{ 2. Formação } \\
\hline \multicolumn{2}{|l|}{ 2.1. Formação Básica } \\
\hline \multicolumn{2}{|l|}{ 2.2. Formação Universitária } \\
\hline 3. Atividades de Formação & \\
\hline
\end{tabular}


Schweiger, K. - Zur brasilianischen Textart "Memorial Acadêmico"

\subsection{Vergleich der Textteile Akademischer Lebenslauf - Memorial}

Bei einer Gegenüberstellung der Textteile Akademischer Lebenslauf ${ }^{11}-$ Memorial $^{1}$ ergeben sich folgende Unterschiede:

Abb.3

\begin{tabular}{|l|l|}
\hline Memorial & Akademischer Lebenslauf \\
\hline$\bullet$ Persönliche Daten & $\bullet$ Persönliche Daten + Foto \\
• Schulische Bildung (Kindergarten, & \\
Grundschule, weiterführende Schule & \\
$\bullet$ Universitäre Ausbildung & $\bullet$ Universitäre Bildung \\
$\bullet$ Publikationsliste (Artikel, Monografien, & $\bullet$ Publikationsliste (meist nur die wichtigsten \\
Kongressakten etc.) & Veröffentlichungen, eventuell Vorträge) \\
$\bullet$ Projekte & $\bullet$ Projekte \\
$\bullet$ Kongressteilnahmen (aktiv, passiv) & $\bullet$ Drittmitteleinnahmen \\
$\bullet$ Betreute Arbeiten (Abschlussarbeiten, & $\bullet$ Mitgliedschaft in \\
Magister-Doktorarbeiten) & Vereinigungen/Verbänden \\
$\bullet$,Zwischen den Zeilen“ & $\bullet$ Gutachtertätigkeiten \\
\hline
\end{tabular}

11 Bei den akademischen Lebensläufen handelt es sich um drei Texte der Bereiche Wirtschaft, Ethik und Anglistik. M. E. ist der akademische Lebenslauf eine relativ fachunabhängige Textart, sodass nicht darauf geachtet wurde, dass die Lebensläufe nur aus der sprachwissenschaftlichen Disziplin stammen. Die Lebensläufe sind im Internet abrufbar (s. Literaturverzeichnis). 


\section{Schweiger, K. - Zur brasilianischen Textart "Memorial Acadêmico"}

Unterschiede hinsichtlich der Textteile sind im Bereich der schulischen Bildung zu finden, die beim Akademischen Lebenslauf nicht ins Gewicht fällt - höchstens wird noch das Abiturjahr genannt.

Die signifikantesten Divergenzen sind sowohl bei der Drittmittelbeschaffung zu finden als auch bei den Kongressteilnahmen. Bei der chronischen Unterfinanzierung deutscher Universitäten spielt die Drittmittelbeschaffung eine große Rolle - nicht zuletzt ein Einstellungskriterium. Verfügt die Person über Kontakte, finanzielle Mittel, um das Forschungsprojekt zu finanzieren bzw. zu beschaffen?

Hinsichtlich der Kongressteilnahmen ist zu bemerken, dass jegliche Art der Teilnahme im Memorial zu verzeichnen ist. Kongressteilnahmen - auch in aktiver Sicht (gehaltene Vorträge) - werden allerdings in einem akademischen Lebenslauf nicht gleichermaßen honoriert wie im Memorial.

Ein weiterer Unterschied besteht in der Auflistung der betreuten Arbeiten. Sicherlich spielt hierbei das Betreuungsverhältnis zwischen Dozent und Student eine Rolle. In Brasilien ist das Verhältnis 12:1. Auf einen Dozenten kommen 12 Studenten (DAAD BERICHTE DER AußENSTELLEN 2006). Für Deutschland ist die Zahl mal drei zu nehmen ${ }^{12}$. Die Auflistung sämtlicher betreuter Arbeiten (z.B. Magisterarbeiten) würde den Rahmen eines Lebenslaufes sprengen.

Des Weiteren scheinen die Mitgliedschaft in Verbänden und wissenschaftlichen Vereinigungen in Deutschland von größerer Bedeutung zu sein. Wohingegen außercurriculare Interessen in den akademischen Lebensläufen nicht zu finden sind und im Memorial Acadêmico als Teil der ganzheitlichen Bildung und Entwicklung des Individuums aufgeführt werden. Bezeichnend dazu ein Kommentar der Deutschen Universitätszeitschrift (2007) über die zehn Sünden der akademischen Bewerbung, wo es heißt: „Als Hobby Marathon oder Blumenstecken angeben: Damit verstoßen Sie gegen das Gesetz: Das Leben findet am Schreibtisch beziehungsweise im Labor statt. Zumindest muss es danach aussehen“.

In den folgenden Abschnitten wird nun näher auf die sprachlichen Realisierungen des Memorial Acadêmicos eingegangen.

\footnotetext{
${ }^{12}$ Als Beispiel sei hier die Betriebswirtschafliche Fakultät der Uni München (LMU) angeführt. Hier kommen 50 Studenten auf einen Professor (http://www.sueddeutsche.de/jobkarriere/16/335865/text/ Zugang:13.06.2007).
} 
Schweiger, K. - Zur brasilianischen Textart "Memorial Acadêmico"

\section{Beschreibung des Memorial Acadêmico}

\subsection{Sprache und Stil}

Bei den Kapitelüberschriften bzw. der Gliederung des Memorials zeichnet sich ein ziemlich heterogenes Bild ab. So finden sich einige „blumige“ Kapitelbeschreibungen, die besonders das poetische Element des Memorials verdeutlichen. Sprichwörter, Wortspielereien, Fragmente der Märchen- und Mythenwelt kennzeichnen dabei das brasilianische Ausdruckssortiment, das sich nicht nur in den Kapitelüberschriften zeigt, sondern sich durch den ganzen Text zieht. Die Sprache in Brasilien, so SCHRÖDER (2003: 174ff), hat zugleich eine Bühnenfunktion, ein „,zur Schau stellen“ von Befindlichkeiten und Meinungen. Pathos, Dramatik und Lebensweisheiten finden sich dabei genauso wieder, wie das Anführen von geflügelten Worten. Nicht zuletzt werden auch häufig Metaphern eingesetzt, meistens aus der Liebes-und Körperwelt -, um die Arbeitswelt zu beschreiben (SCHRÖDER 2003: 198ff).

In den vorliegenden Memoriais lassen sich diese von SCHRÖDER aufgezählten Ausdrucksweisen wiederfinden (s. Beispiele 5-14).

\section{a) Metaphorische Ausdrucksweisen (Liebesmetaphern)}

\section{Beispiel 5}

Foi lá também que me apaixonei pela análise sintática. E, infelizmente, foi também lá que desisti de entender a matemática.

Dort habe ich mich in die Satzanalyse verliebt. Und leider habe ich es auch dort aufgegeben, Mathematik zu verstehen.

\section{Beispiel 6}

Fui apresentada à literatura francesa que me seduziu tanto quanto a brasileira e a portuguesa.

Ich wurde der französischen Literatur vorgestellt, die mich so verführt hat wie die brasilianische und die portugiesische. 
Schweiger, K. - Zur brasilianischen Textart "Memorial Acadêmico"

\section{Beispiel 7}

Como num casamento, meu relacionamento com esse período tem muito de amor...e um pouco de ódio!

Wie in einer Ehe gibt es in meiner Beziehung zu dieser Epoche viel Liebe ... und ein bisschen Hass.

\section{Beispiel $\mathbf{8}^{13}$}

Anos de Purgatório. A faculdade de Direito do Largo de São Francisco.

Die Jahre des Fegefeuers.Die juristische Fakultät Largo de São Francisco.

\section{b) Wortspielereien, Weisheiten}

\section{Beispiel 9}

Quando uma porta se fecha...outra se abre?

Wenn eine Tür sich schließt... öffnet sich eine andere?

\section{Beispiel 10}

Publicar é preciso. Viver não é preciso?

Veröffentlichen ist notwendig. Leben ist nicht notwendig?

\section{Beispiel 11}

Mas, como diria o finado Brás Cubas: „não adiantemos os sucessos”.

Aber wie sagte der gute Brás Cubas: „,Führen wir den Erfolg nicht zu früh herbei“".

\section{Beispiel 12}

Assim prosseguia, tentando a fome de saber e a de viver...

So kam ich voran, den Wissens- und Lebensdurst ausprobierend...

\section{Beispiel 13}

Aprovada...fui matriculada.

Prüfung bestanden... eingeschrieben.

${ }^{13}$ Beispiel 6, 7, 11 und 14 sind Kapitelüberschriften. 
Schweiger, K. - Zur brasilianischen Textart "Memorial Acadêmico"

\section{Beispiel 14}

Orientandos, orientados e desorientados.

Betreut Werdende, Betreute und Orientierungslose.

\section{c) Dramatik}

\section{Beispiel 15}

Tenho feito o máximo esforço para ser honesto e fiel ao roteiro da minha carreira universitária.

Ich habe mich maximal bemüht, ehrlich und treu meine akademische Laufbahn zu verfolgen.

\section{Beispiel 16}

Os anos que estudei na fundação ficarão registrados para sempre na minha memória como anos difíceis [...] mas também são lembrados como momentos repletos de saber, companheirismo, amizade, compreensão e, principalmente, de generosidade.

Die Jahre, die ich an der Schule gelernt habe, werden mir immer als schwierige Jahre in Erinnerung bleiben [...], aber auch als Momente voller Wissen, Kameradschaft, Freundschaft, Verständnis und v.a. Großzügigkeit.

\subsection{Stimulierende und deliberative Sprechhandlungen}

Der Terminus "Stimulierende Texthandlungen” wurde dem der „stimulierenden Textmerkmale“ von GROEBER (1982:212) vorgezogen, um den Handlungscharakter von Sprache zu verdeutlichen. Unter diesem Begriff sind Fragen bzw. rhetorische Fragen, elliptische Äußerungen, persönliche Worte, Markierungen/Hervorhebung bestimmter Textteile durch den Autor gemeint und verdeutlichen die Unmittelbarkeit und Direktheit, mit der sich der Autor an den Leser wendet. Nicht zuletzt erzeugen sie den Eindruck von Dialogizität zwischen Autor und Leser. Dazu tragen vor allem die Klammersetzung als auch die Auslassungspunkte bei, die den Eindruck der Mündlichkeit verstärken (SACHTLEBER 


\section{Schweiger, K. - Zur brasilianischen Textart "Memorial Acadêmico"}

1993, 182). Des Weiteren zähle ich zu den stimulierenden Texthandlungen auch deliberative ${ }^{14}$ Texthandlungen. Unter deliberative Frage wird das „Nach-außen-bringen“ eines Reflexionsprozesses verstanden (ZIFONUN ET AL. 1997: 115). „Die deliberative Frage hat den Zweck, eine Problemlösung dadurch zu befördern, dass das Problem (...) ,nach außen“ (...) gesetzt wird und in dieser Formulierung einer mentalen Bearbeitung durch den Sprecher selbst und/oder den Hörer zugänglich gemacht wird (....“. In den folgenden Beispielen zeigt sich, wie hier versucht wird, den anderen an seinem Seelenzustand teilnehmen zu lassen, um ihm Einsicht in das Innenleben des Verfassers zu gewähren. Dem Leser wird die Möglichkeit gegeben, an Entscheidungsprozessen bzw. Reflexionen des Verfassers teilzunehmen.

\section{a) Deliberative Sprechhandlungen}

\section{Beispiel 17}

Estudando-se as crônicas, estabelece-se o impasse qual será sua verdadeira função: informar? interpretar (...)?

Bei der Analyse von Chroniken gerät man in einen Engpass: Welche wird wohl ihre wahre Funktion sein? Zu informieren? Zu interpretieren?

\section{Beispiel 18}

A partir dessa síntese, me interrogo: como operar com os conceitos de quantidade e qualidade? Como medir em número os conceitos de quantidade $\mathrm{e}$ qualidade? Como medir em número de páginas o esforço, o tempo do prazer na tradução do verso, para tentar recuperar em nossa língua algo da beleza original de um poema?

Von dieser Synthese ausgehend habe ich mich gefragt: Wie gehe ich mit den Ideen/Konzepten der Quantität und Qualität um? Wie messe ich die Zahl der Seiten, die Anstrengung, die Zeit des Vergnügens mit der Übersetzung von Versen, um in unserer Sprache diese ursprüngliche Schönheit des Gedichts einzufangen?

\section{Beispiel 19}

Voltando às origens, encontro-me agora, depois de quase três anos de Alemanha, em um país do qual já não me lembrava muito. Quanta diferença! E o que fazer?

\footnotetext{
${ }^{14}$ EHLICH/REHBEIN (1997) verwenden den Begriff der Exothese (s. ZIFONUN 1997 et al.). Man könnte daher auch von exothetisierenden Sprechhandlungen sprechen.
} 
Schweiger, K. - Zur brasilianischen Textart "Memorial Acadêmico"

Zurück zu den Wurzeln, ich befinde mich jetzt, nach fast drei Jahren in Deutschland, in einem Land, an das ich mich kaum noch erinnere, was für ein Unterschied! Und was mache ich jetzt?

\section{Beispiel 20}

Como lidar com o poder? Bancas e Comissões.

Wie geht man mit der Macht um? Auswahlkommitees und Prüfungskommissionen.

\section{b) Klammersetzung, Ausrufe und Auslassungspunkte}

\section{Beispiel 21}

O ano 1951 foi decisivo na minha vida escolar. Por alguma razão misteriosa que não consigo identificar, transformei-me de aluno rebelde (para os padrões da época, por favor!) em aluno bem comportado.

Das Jahr 1951 war ein entscheidendes Jahr in meiner Schullaufbahn. Aus irgendeinem mysteriösen Grund, den ich nicht ausmachen kann, verwandelte ich mich vom rebellischen Schüler (aber im Rahmen dieser Zeit, bitte schön!) in einen braven Schüler.

\section{Beispiel 22}

Que bom que ainda não tínhamos filhos!

Wie gut, dass wir noch keine Kinder hatten!

\section{Beispiel 23}

Início de 1976, lá estou eu prestando vestibular CESEA. Consegui...Português e Alemão!

Anfang 1976, da bin ich nun und absolviere die Zulassungsprüfung CESEA. Ich habe es geschafft...für Portugiesisch und Deutsch!

\section{Beispiel 24}

Também houve um concurso para historiográfico no Museu Paulista, com 80 candidatos e duas vagas. Adivinhem em que lugar fiquei! Em terceiro, é claro! Es gab auch ein Bewerbungsverfahren als Geschichtswissenschaftler am Paulista Museum mit 80 Bewerbern und zwei Stellen. Ratet mal auf welchen Platz ich kam! Auf den dritten, natürlich! 


\section{Schweiger, K. - Zur brasilianischen Textart "Memorial Acadêmico"}

\subsection{Metakommunikative Äußerungen}

Unter „Metakommunikation“ subsumieren sich diejenigen Äußerungen, bei denen der Autor sich direkt an den Leser wendet und aus dem Text „heraustritt“, um dem potenziellen Leser über Schwierigkeiten, Probleme, Unsicherheiten oder sonstige Unwäglichkeiten beim Verfassen des Textes $\mathrm{zu}$ berichten. „Metakommunikative Äußerungen können als Sprechhandlungen betrachtet werden, die die laufende Kommunikation zum Gegenstand haben und deren wesentliches Merkmal ihr reflexiver, d.h. rückbezüglicher Charakter ist" (KAISER 2002: 248).

RADEMACHER (1998:45ff) macht zwei Hauptfunktionen metakommunikativer Äußerungen aus: eine interaktive Funktion und eine kommunikative Funktion. Erstere ist zuständig für die Regulierung sozialer Nähe und Distanz letztere dient der Organisation des Dialogs; der Vermeidung von Missverständnissen und der allgemeinen Verständnissicherung.

Diese metakommunikativen Äußerungen finden sich vielmals in dem untersuchten Korpus, dies mag zum einen daran liegen, dass ein memorial acadêmico keine gängige Textart ist, d.h., man schreibt diese selten mehr als einmal bzw. fügt ansonsten nur etwas hinzu oder ändert ab. Deshalb fühlen sich manche unsicher, vielleicht sogar überfordert und bringen dies „,nach außen“ (s. Bsp. 25-26):

\section{Beispiel 25}

Habituada à objetividade, à clareza, à logica dominantes no discurso do poder absoluto do aprendizado universitário, confesso que não me sinto à vontade com o uso da primeira pessoa, condição básica para a elaboração de um memorial.

An die im akademischen Diskurs vorherrschende Objektivität, Klarheit und Logik gewöhnt, bekenne ich, dass ich mich nicht wohl fühle beim Gebrauch der ersten Person - Grundvoraussetzung zum Verfassen eines Memorials

\section{Beispiel 26}

Síntese impossível: sob essas divisas este memorial foi redigido, menos por opção do autor que pela inevitabilidade desses conceitos.

Unmögliche Synthese: Unter diesen Umständen wurde das Memorial verfasst, weniger aufgrund der Wahl des Autors vielmehr wegen der Unvermeidlichkeit dieser Konzepte. 
Schweiger, K. - Zur brasilianischen Textart "Memorial Acadêmico"

\subsection{Zum illokutiven Gehalt der Äußerungen}

In den bisher vorgestellten Beispielen zeigt sich, dass auf der Ebene der illokutiven Akte neben den rein assertiven oder auch repräsentativen Sprechhandlungen, denen die Informationsvermittlung zugrunde liegt, sich auch argumentative wie auch deskriptive Sprechhandlungen (Beispiel 27-28 z.B. genaue Deskription der Umgebung) wiederfinden.

\section{Beispiel 27}

Santo André era uma cidade tranquila e pacata. Santo André war eine ruhige und friedvolle Stadt.

\section{Beispiel 28}

Era uma escola totalmente diferente da anterior. Construida em madeira - com exceção dos três banheiros de dois metros quadrados cada um (masculino, feminino e das professoras) e da cozinha que eram de alvenaria - possuia apenas três salas e uma para a Direção.

Die Schule war ganz anders als die vorherige Schule. Aus Holz gebaut - mit Ausnahme der drei Toiletten mit jeweils 2 Quadratmetern Fläche (eine für Schüler, eine für Schülerinnen und die Toilette der Lehrerinnen) und der Küche, die aus Ziegeln.gebaut waren - hatte die Schule nur drei Klassenzimmer, und einen Raum für die Direktion.

Besonders charakteristisch sind die rechtfertigenden und bewertenden Sprechhandlungen, die in der Form in einem akademischen Lebenslauf nicht anzutreffen sind. Es ist im Memorial möglich, über die „negativen Seiten“ des Curriculum vitae zu schreiben, über die verpassten Chancen, Umwege, falsche Entscheidungen. „Ausrutscher“ sind erlaubt - wohingegen der akademische Lebenslauf gefälligst lückenlos zu sein hat. So auch das folgende Beispiel:

\section{Beispiel 29}

Há sempre um deslizamento", diz Marguerite Yourcenar em "De olhos abertos" e é esse deslizamento intelectual que tentarei mostrar e justificar aqui. Es gibt immer ein Abdriften, sagt Marguerite Yourcenar in "Mit offenen Augen/Eyes wide shut" und dieses intellektuelle Abdriften versuchte ich, zu zeigen und zu rechtfertigen. 
Schweiger, K. - Zur brasilianischen Textart "Memorial Acadêmico"

\subsection{Die Konstituente "Familie"}

In den Memoriais ist generell auch ein stärkerer Bezug zur Familie auszumachen. Wie die Beispiele 30 und 31 zeigen:

\section{Beispiel 30}

Minha avó era muito querida por mim, ensinava-me francês, latim, e boas maneiras. Aprendi com ela a gostar de perfumes.

Ich mochte meine Oma sehr, sie hat mir Französisch, Latein und gutes Benehmen beigebracht. Ich lernte von ihr auch Parfüm zu mögen.

\section{Beispiel 31}

Das minhas tias.herdei o gosto pela docência.

Von meinen Tanten habe ich die Lust am Unterrichten geerbt.

Einer der Gründe weshalb die Familie in Brasilien eine tendenziell höhere Wertigkeit besitzt, könnte laut GÜSS (1998) in der Individualismus-Kollektivismus-Theorie HoFSTEDES (1980) ihre Erklärung finden. Mit der Familie werden Schutz und Sicherheit verbunden, gerade dann auch, wenn ein „starker“ Staat fehlt (z.B: i.S. eines Sozialstaates). Wichtige Entscheidungen werden - so auch die berufliche Laufbahn - zusammen bzw. von der Familie gefällt, da dieser bei der Finanzierung des Studiums eine wichtige Rolle zufällt. Die Bindung zu den Eltern in Brasilien ist generell etwas enger und es werden soziale Errungenschaften in diesem Zusammenhang mit Stolz hervorgehoben.

\section{Erklärungsversuch aus kulturhistorischer Perspektive}

Die bisher gezeigten Beispiele weisen einen besonders auffallend hohen Anteil an direkten Redeelementen auf; man hat das Gefühl man steht in direktem Kontakt mit dem Autor. Gerade die deliberativen Texthandlungen wie auch die metakommunikativen Äußerungen vermitteln den Eindruck einer dialogischen Gesprächssituation. SCHRÖDER (2003), die Brasilianer und Deutsche zu verschiedensten Lebenskonzepten (Arbeit, Familie, 


\section{Schweiger, K. - Zur brasilianischen Textart "Memorial Acadêmico"}

Liebe, Zukunft etc.) in einem kulturkontrastiven Vergleich interviewt hat, stellt bei den Brasilianern einen von Oralität geprägten Erzählstil fest. In einem von ihr (SCHRÖDER 2003: 136) geführten Interview mit der Kulturforscherin DiLMA DE MELO Silva äußert sich diese zum Erzählstil der Brasilianer folgendermaßen:

O que marca a nossa cultura nesse sentido é, sem dúvida, a oralidade. Isto implica, o que marca uma conversa aqui, no presente momento, é contar histórias, uma expressão extremamente narrativa. E a história nunca é contada diretamente. Não é dita com uma palavra, sempre com uma história, um provérbio. É mais um cenário.

Was unsere Kultur in diesem Sinn auszeichnet, ist zweifelsohne die Oralität. Das impliziert, dass das, was eine Konversation in einem bestimmten Moment ausmacht, das Geschichtenerzählen, eine extrem narrative Ausdrucksweise ist. Und die Geschichte wird niemals direkt erzählt. Sie wird nicht mit einem Wort erzählt, immer mit einer kleinen Anekdote dabei, mit einem Sprichwort. Es ist eher ein Szenario.

Begründet wird dies durch die langjährige Unterdrückung durch die portugiesischen Eroberer. Mehrere Jahrhunderte lang war es den Brasilianern nicht möglich bzw. verboten, Universitäten zu gründen, Bücher zu erwerben etc. So schreibt AZZI (2005), dass während der Kolonialzeit das Schrifttum verboten wurde und die Verbreitung von Schriften daher sehr beschränkt war, wenn überhaupt vorhanden, mussten diese von der weit entfernten Stadt bestellt werden. Die Missionierung der luso-brasilianischen Gesellschaft und Verbreitung der theologischen Wahrheiten erfolgte so mündlich oder durch Imagination. Ein nicht unbeachtlicher Teil der katholischen Doktrin wurde durch Statuen, Malerei, rituelle Gesten, Gesänge und religiöse Inszenierungen vermittelt. Aufgrund der hier genannten kulturhistorischen Gegebenheiten, zeigt sich, dass sich in Brasilien eine stärker oralisierte Kultur- und Kommunikationsgemeinschaft herausgebildet hat, deren Charakteristika sich noch in den heutigen Text- und Diskursarten niederschlagen bzw. noch anzutreffen sind.

\section{Literaturverzeichnis}

AzZI, R. A teologia católica na formação da sociedade colonial brasilieira. Petrópolis/ RJ, Editora vozes, 2005. 


\section{Schweiger, K. - Zur brasilianischen Textart "Memorial Acadêmico"}

Bruss, E. Die Autobiographie als literarischer Akt. In: NigGL, G. (Hrsg.) Die Autobiographie. Zur Form und Geschichte einer literarischen Gattung. Darmstadt, Wissenschaftliche Buchgesellschaft, 1989, 33-55.

DAAD. DAAD-Berichte der Außenstellen. Deutsch Akademischer Austauschdienst, Bonn, 2006.

EhLICH, K. Textsorten - Überlegungen zur Praxis der Kategoriebildung in der Textlinguistik. In: MACKELDEY, R. (Hrsg.) Textsorten/Textmuster in der Sprech- und Schriftkommunikation. Leipzig, 1990, 17-30.

ESSER, R. Etwas ist mir geheim geblieben am deutschen Referat. Kulturelle Geprägtheit wissenschaftlicher Textproduktion und ihre Konsequenzen für den universitären Unterricht von Deutsch als Fremdsprache. München, iudicium, 1997.

Güss, D. Spontanität versus Gründlichkeit: Eine Untersuchung über das Planen in Brasilien und Deutschland. Memorandum Nr. 26, Otto-Friedrich Universität Bamberg, Lehrstuhl Psychologie, 1998.

Hofstede, G. Culture's consequences: International differences in work-related values. Beverly-Hills, Sage, 1980.

Deutsche Universitätszeitschrift www.duz.de/docs/stellenthemen/bewerbungssuenden.html. Zugang: 20.04.07

KAISER, D. Wege zum wissenschaftlichen Schreiben - Eine kontrastive Untersuchung zu studentischen Texten aus Venezuela und Deutschland. Tübingen, Stauffenburg, 2002.

Lehmann, J. Bekennen - Erzählen - Berichten. Studien zur Theorie und Geschichte der Autobiographie. Tübingen, Niemeyer, 1988.

Misch, G. Begriff und Ursprung der Autobiographie. In: NigGL, G. (Hrsg.) Die Autobiographie. Zur Form und Geschichte einer literarischen Gattung. Darmstadt, Wissenschaftliche Buchgesellschaft, 1989, 33-55.

Prado Toledo, G.D. V. / Soligo, R. Memorial de Formação - quando as memórias narram a história da formação. In: DERS. (Hrsg.) Porque escrever é fazer história. 2 ed. Campinas, Graf. FE, v.1/2005, 47-52.

Ramos, M. A. / GonCAlves, R. E. As narrativas autobiográficas do professor como estratégia de desenvolvimento e prática da supervisão. In: Formação reflexiva de professores estratégias de supervisão. Alacrão, Isabel Org. Editora Porto, 1996.

RADEMACHER, U. Metakommunikation. Das Sprechen über das Sprechen aus sozialpsychologischer Perspektive. Heidelberg, Univ. Diss., 1998.

REHBEIN, J. Einführung. In: DERS. (Hrsg.) Interkulturelle Kommunikation. Tübingen, Narr, 1985, 7-32.

SACHTLEBER, S. Die Organisation wissenschaftlicher Texte: eine kontrastive Analyse. Frankfurt, Lang, 1993.

SCHLIEBEN-LANGE, B. Metasprache und Metakommunikation. Zur Überführung eines sprachphilosophischen Problems in die Sprachtheorie und in die sprachwissenschaftliche Forschungspraxis“. In: DERS. (Hrsg.) Sprachtheorie. Hamburg, Hoffmann \& Campe, 1975, 189-205. 


$$
\text { Schweiger, K. - Zur brasilianischen Textart "Memorial Acadêmico" }
$$

SCHRÖDER, U. Brasilianische und deutsche Wirklichkeit. Eine vergleichende Fallstudie zu kommunikativ erzeugten Sinnwelten. Wiesbaden, Deutscher Universitätsverlag, 2003.

StEPHAN, A. Autobiographien, Memoiren und Oral-History als historische Quellen. In: (o.A.V.): Digitales Handbuch zur Geschichte und Kultur Russlands und Osteuropas.www.vifaost.de, 2004.

http://www.sueddeutsche.de/jobkarriere/16/335865/text/ Zugang: 13.06.2007

WAizBORT, L. Para uma sociologia do memorial acadêmico - um fragmento. In: Revista de Teoria Literária e Literatura Comparada, São Paulo 1998, v. 3, 77-82.

ZIFOnUn, G./ HofFMAnN, L. /STRECKER, B. ET AL. (Hrsg.) Grammatik der deutschen Sprache. Bd. 1 Sprache und Illokution. Berlin, de Gruyter, 1997.

\section{Akademische Lebensläufe}

http://www.ebs.edu/fileadmin/redakteur/faculty/CV/CV_Moeller_Sabine.pdf. (Zugang: 19.08.2009)

http://www.ethik.uni-jena.de/de/images/stories/personal/2/Lebenslauf.pdf (Zugang: 19.08.2009)

http://www.unikonstanz.de/FuF/Philo/LitWiss/angame/images/content/Rosenthal_Lebenslauf _2008-05-26.pdf (Zugang: 19.08.2009) 